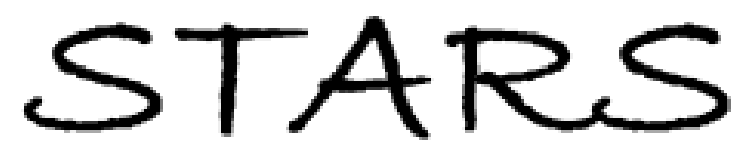

University of Central Florida

STARS

$1-1-2014$

\title{
Failure of semiclassical models to describe resistivity of nanometric, polycrystalline tungsten films
}

Dooho Choi

Xuan Liu

Patrick K, Schelling

University of Central Florida

Kevin R. Coffey

University of Central Florida

Katayun Barmak

Find similar works at: https://stars.library.ucf.edu/facultybib2010

University of Central Florida Libraries http://library.ucf.edu

This Article is brought to you for free and open access by the Faculty Bibliography at STARS. It has been accepted for inclusion in Faculty Bibliography 2010 s by an authorized administrator of STARS. For more information, please contactSTARS@ucf.edu.

\section{Recommended Citation}

Choi, Dooho; Liu, Xuan; Schelling, Patrick K.,; Coffey, Kevin R.; and Barmak, Katayun, "Failure of semiclassical models to describe resistivity of nanometric, polycrystalline tungsten films" (2014). Faculty Bibliography 2010s. 5182.

https://stars.library.ucf.edu/facultybib2010/5182

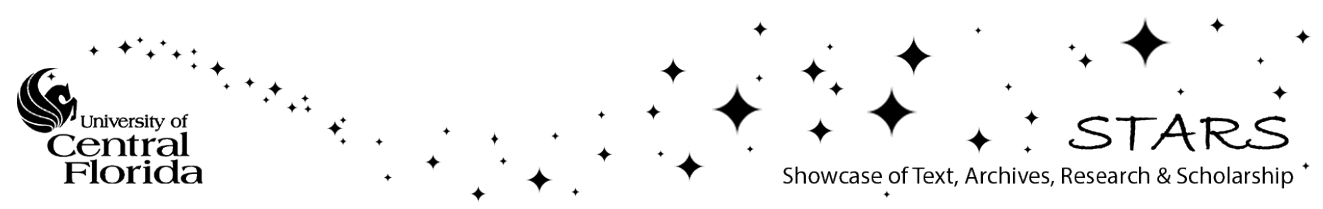




\section{Failure of semiclassical models to describe resistivity of nanometric, polycrystalline tungsten films}

Cite as: J. Appl. Phys. 115, 104308 (2014); https://doi.org/10.1063/1.4868093

Submitted: 12 November 2013 . Accepted: 26 February 2014 . Published Online: 13 March 2014

Dooho Choi, Xuan Liu, Patrick K. Schelling, Kevin R. Coffey, and Katayun Barmak
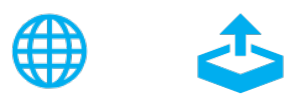

View Online

Export Citation

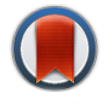

CrossMark

\section{ARTICLES YOU MAY BE INTERESTED IN}

Electron mean free path in elemental metals

Journal of Applied Physics 119, 085101 (2016); https://doi.org/10.1063/1.4942216

Phase, grain structure, stress, and resistivity of sputter-deposited tungsten films

Journal of Vacuum Science \& Technology A 29, 051512 (2011); https://doi.org/10.1116/1.3622619

Size effects and charge transport in metals: Quantum theory of the resistivity of nanometric metallic structures arising from electron scattering by grain boundaries and by rough surfaces Applied Physics Reviews 4, 011102 (2017); https://doi.org/10.1063/1.4974032

\section{Applied Physics Reviews} Now accepting original research

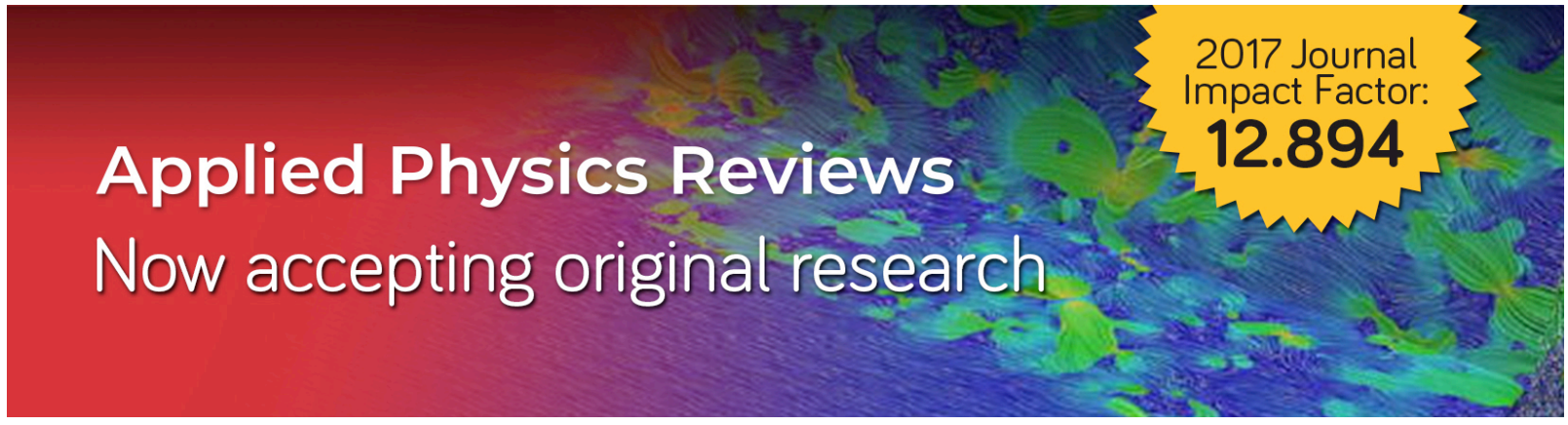




\title{
Failure of semiclassical models to describe resistivity of nanometric, polycrystalline tungsten films
}

\author{
Dooho Choi, ${ }^{1,2}$ Xuan Liu, ${ }^{1}$ Patrick K. Schelling ${ }^{3}$ Kevin R. Coffey, ${ }^{4}$ and Katayun Barmak ${ }^{1,5}$ \\ ${ }^{1}$ Department of Materials Science and Engineering, Carnegie Mellon University, 5000 Forbes Avenue, \\ Pittsburgh, Pennsylvania 15213, USA \\ ${ }^{2}$ Korea Railroad Research Institute, 360-1 Woulam, Uiwang, Kyunggi 437-757, South Korea \\ ${ }^{3}$ Advanced Materials Processing and Analysis Center and Department of Physics, University of Central Florida, \\ 4000 Central Florida Boulevard, Orlando, Florida 32816, USA \\ ${ }^{4}$ Department of Materials Science and Engineering, University of Central Florida, 4000 Central Florida \\ Boulevard, Orlando, Florida 32816, USA \\ ${ }^{5}$ Department of Applied Physics and Applied Mathematics, Columbia University, 500 West 120th Street, \\ New York, New York 10027, USA
}

(Received 12 November 2013; accepted 26 February 2014; published online 13 March 2014)

\begin{abstract}
The impact of electron scattering at surfaces and grain boundaries in nanometric polycrystalline tungsten (W) films was studied. A series of polycrystalline W films ranging in thickness from 10 to $310 \mathrm{~nm}$ and lateral grain size from 74 to $133 \mathrm{~nm}$ were prepared on thermally oxidized Si. The Fuchs-Sondheimer surface-scattering model and Mayadas-Shatzkes grain-boundary scattering model were employed for quantitative analyses. Predictions from the theoretical models were found to deviate systematically from the experimental data. Possible reasons for the failure of the theoretical models to describe the experimental data are explored. Finally, a discussion of the crucial features lacking from existing models is presented, along with possible avenues for improving the models to result in better agreement with experimental data. (C) 2014 AIP Publishing LLC. [http://dx.doi.org/10.1063/1.4868093]
\end{abstract}

\section{INTRODUCTION}

Tungsten (W) has been recently considered as a potential candidate to replace $\mathrm{Cu}$ for future semiconductor interconnects. ${ }^{1-3}$ The replacement is predicted to improve the interconnect reliability due to stronger W-W bonding, as reflected by the very high melting point of $3695 \mathrm{~K}$ compared with $\mathrm{Cu}$ at $1357 \mathrm{~K}{ }^{4}$ Further, the electron mean free path (EMFP) of $\mathrm{W}$ at room temperature, ${ }^{2} 19.1 \mathrm{~nm}$, is less than half that of $\mathrm{Cu}, 39 \mathrm{~nm}$; hence, a reduction in the impact of resistivity size effect is expected. However, the higher room temperature bulk resistivity of $\mathrm{W}$ at $5.3 \mu \Omega \mathrm{cm}$, compared to $\mathrm{Cu}$ at $1.7 \mu \Omega \mathrm{cm}$, requires quantitative knowledge of the grain boundary and surface scattering contributions to resistivity size effect of $\mathrm{W}$ with decreasing conductor dimensions, e.g., line-height, line-width and grain size, to assess whether $\mathrm{W}$ may provide lower resistivity as a nanoscale interconnect. This knowledge can, in part, be obtained experimentally, but a sound theoretical understanding is also needed to allow extrapolation beyond a single data set and to dimensions smaller than currently examined.

The resistivity size effect is typically attributed to electron momentum loss along the direction of current flow due to surface and grain boundary scattering. ${ }^{5}$ The commonly used theoretical models for these scattering mechanisms are the Fuchs-Sondheimer (FS) surface scattering model ${ }^{6,7}$ and the Mayadas-Shatzkes (MS) grain boundary scattering model. ${ }^{8}$ The FS model incorporates a specularity parameter $(p)$, the fraction of electrons that are specularly scattered from the surfaces, with $(1-p)$ denoting the fraction scattered diffusely. ${ }^{6,7}$ The film resistivity of the FS model is computed as
$\rho_{F S}=\rho_{i}\left[1-\left(\frac{3}{2 k}\right)(1-p) \int_{1}^{\infty}\left(\frac{1}{t^{3}}-\frac{1}{t^{5}}\right) \frac{1-\exp (-k t)}{1-p \exp (-k t)} d t\right]^{-1}$,

where $k=\frac{L}{\lambda}$, where $L$ is the film thickness, $\lambda$ is the bulk electron mean-free path, and $\rho_{i}$ is the bulk resistivity of the metal.

The MS model incorporates a reflection coefficient $(R)$ representing the fraction of electrons that are reflected at columnar grain boundaries. ${ }^{8}(1-R)$ then corresponds to the fraction transmitted. In case of films with infinite thickness, i.e., with no interaction between grain boundary scattering and surface scattering, the film resistivity due to grain boundary scattering by the MS model is

$$
\rho_{M S}=\rho_{i}\left[1-\frac{3}{2} \alpha+3 \alpha^{2}-3 \alpha^{3} \ln \left(1+\frac{1}{\alpha}\right)\right]^{-1},
$$

where $\alpha=\left(\frac{\lambda}{g}\right) \frac{R}{1-R}$, and $g$ is in-plane (lateral) grain size of columnar grains. Following Matthiessen's rule, total film resistivity in the regime of size effect is often described with the summation of the contributions of the FS surface scattering and MS grain boundary scattering. This combined model, with no interaction between the two scattering mechanisms, is referred to as "FS + MS" model. MayadasShatzkes also developed a model which allows an interaction between the two scattering mechanisms, where the grain boundary scattering process redirects electrons with initial momentum parallel to the external surfaces 
(i.e., having no possibility of scattering at surfaces) towards the surfaces, which increases resistivity over and above the simple summation of the surface and grain boundary scattering. This model is referred to as "MSS" model, ${ }^{9}$ and the total film resistivity according to this model is computed as

$$
\begin{aligned}
\rho_{M S S}= & {\left[\frac{1}{\rho_{M S}}-\left(\frac{6}{\pi k \rho_{i}}\right)(1-p)\right) \int_{0}^{\pi / 2} d \varphi \int_{1}^{\infty} d t \frac{\cos ^{2} \varphi}{H^{2}(t, \varphi)} } \\
& \left.\times\left(\frac{1}{t^{3}}-\frac{1}{t^{5}}\right) \frac{1-\exp [-k t H(t, \varphi)]}{1-p \exp [-k t H(t, \varphi)]} d t\right]^{-1},
\end{aligned}
$$

where

$$
H(t, \varphi)=1+\frac{\alpha}{\cos \varphi \sqrt{1-\frac{1}{t^{2}}}} .
$$

While studies of the resistivity size effect for $\mathrm{W}$ films and lines have been reported in a number of prior publications, ${ }^{10-14}$ these studies (1) adopted bulk EMFP values from early reports that significantly overestimated

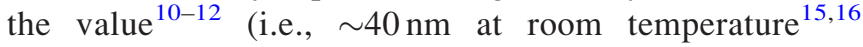
instead of the value of $19.1 \mathrm{~nm}$ determined using density functional theory (DFT) calculations ${ }^{2}$ ), (2) treated EMFP as a fitting parameter in addition to the other fitting parameters $p$ and $R,{ }^{13}$ an approach which was shown to produce a non-unique set of fitting parameters, ${ }^{2}$ (3) introduced an unjustified "dead layer," as thick as $12 \mathrm{~nm}$, to describe the resistivity size effect, ${ }^{12}$ or (4) neglected the role of grain boundary scattering in polycrystalline films. ${ }^{14}$ Due to these shortcomings, it is necessary to re-evaluate the impact of the resistivity size effect in $\mathrm{W}$, aimed at assessing its potential as the next-generation semiconductor interconnect material. In addition, it is of significant fundamental interest to establish how effective existing theoretical models can be expected to describe experimental data, especially in the limit of very thin film thickness and/or low temperatures.

In this study, the role of surface scattering and grain boundary scattering in the resistivity size effect for polycrystalline $\mathrm{W}$ thin films is investigated. The measured film thickness and grain size are in the ranges of 10 to $310 \mathrm{~nm}$ and 74 to $133 \mathrm{~nm}$, respectively. The FS, FS + MS, and MSS models are employed to determine $p$ and $R$ for polycrystalline W films. It is found that the relative contributions of the two scattering mechanisms are similar in both the FS + MS and MSS models. However, it is also found that the theoretical models systematically deviate from the experimental data. A discussion of possible reasons for the inadequacy of the models is presented. These include the possibility of more than one relevant length scale for bulk electron scattering, neglect of electron interference including mini-band formation or electron localization effects, and neglect of size-dependence of the phonon spectrum relevant for electron-phonon scattering. Possible directions for improvement of theoretical models are discussed.

\section{EXPERIMENTS}

Polycrystalline $\mathrm{W}$ films having thicknesses from 10 to $310 \mathrm{~nm}$ were deposited onto thermally oxidized (100)-Si wafers by DC magnetron sputtering using a $99.95 \%$ purity $\mathrm{W}$ target. The deposition temperature and sputtering power were $520{ }^{\circ} \mathrm{C}$ and $250 \mathrm{~W}$, respectively, at which the deposition rate was $1.4 \AA / \mathrm{s}$. Following deposition, the films were annealed ex-situ at $850{ }^{\circ} \mathrm{C}$ for $2 \mathrm{~h}$ in $\mathrm{Ar}+4 \% \mathrm{H}_{2}$ ambient. Additional details of the film processing conditions can be found elsewhere. ${ }^{1-3}$

$\theta-2 \theta$ x-ray diffraction (XRD) scans were performed using $\mathrm{Cu} K \alpha$ radiation. In order to suppress the strong $\mathrm{Si}$ (400) peak near $69.2^{\circ}$ without significantly affecting the intensity of $\mathrm{W}$ peaks, samples were omega-tilted by $6.5^{\circ} .{ }^{1}$ $\mathrm{X}$-ray $\psi$-scans were also performed to identify the preferred crystallographic orientation of the grains in the films. The film thicknesses were measured using $\mathrm{x}$-ray reflectivity (XRR). All x-ray patterns were obtained on the PANalytical PW 3040/60 instrument. Electrical resistivities of the films were measured using the van der Pauw method. ${ }^{17}$

For the grain size measurement, a transmission electron microscope (TEM) based orientation mapping technique was employed. ${ }^{18-21}$ About 20 orientation maps were recorded using the orientation mapping system (NanoMEGAS) installed on an FEI Tecnai F20 TEM (FEI Corporation) with a field emission gun and an accelerating voltage of $200 \mathrm{kV}$. The maps were then analyzed using TSL OIM software (EDAX) in a similar manner as described elsewhere. ${ }^{21}$ Grain boundary networks were reconstructed using the TSL OIM software, based on which the lateral average grain size in the films was determined. The reported grain size is the equivalent circle diameter of the mean area for a statistically significant number of grains (of the order of 1000 per sample), using the relation

$$
\text { Grain size }=\sqrt{\frac{4 \bar{A}}{\pi}}
$$

where $\bar{A}$ is the mean area. The errors on these diameters are quoted as $\pm 2 \sigma$ values at a confidence level of $95 \%$ for the given grain population. ${ }^{22}$ Table I summarizes film identification, thickness, resistivity at $293 \mathrm{~K}$ and at $4.2 \mathrm{~K}$, lateral grain size, number of grains measured, and the ratio of grain size to film thickness.

\section{RESULTS AND DISCUSSION}

Figure 1(a) gives a representative $\theta-2 \theta$ XRD scan, conducted for film $\mathrm{G}$. The various Bragg-reflected peaks confirm that the film is polycrystalline, in the bcc $\alpha$-phase. ${ }^{1}$ No highresistivity A15 $\beta$-phase was observed. A $\psi$-scan for film G with respect to the (110) peak is also given in Fig. 1(b), where the relatively small peak to valley ratio (around two) and the presence of the additional $\{110\}$ peak near $60^{\circ}$ indicate that the polycrystalline film is weakly (110) fibertextured.

Figure 2(a) shows a representative crystal orientation map for film $\mathrm{F}$ from which lateral grain size is measured as described earlier. The cross-sectional TEM dark-field 
TABLE I. Film identification, thickness, resistivity at $293 \mathrm{~K}$ and $4.2 \mathrm{~K}$, lateral grain size, number of grains measured, and ratio of grain size to film thickness.

\begin{tabular}{|c|c|c|c|c|c|c|}
\hline \multirow[b]{2}{*}{ Film ID } & \multirow[b]{2}{*}{ Thickness (nm) } & \multicolumn{2}{|c|}{ Resistivity $(\mu \Omega \mathrm{cm})$} & \multirow[b]{2}{*}{ Grain size $(\mathrm{nm})$} & \multirow[b]{2}{*}{ Number of grains measured } & \multirow[b]{2}{*}{ Ratio } \\
\hline & & $293 \mathrm{~K}$ & $4.2 \mathrm{~K}$ & & & \\
\hline A & 10.3 & 14.4 & 8.5 & $74 \pm 4$ & 1270 & 7.2 \\
\hline $\mathrm{B}$ & 20.6 & 11.0 & 5.7 & $90 \pm 5$ & 1230 & 4.4 \\
\hline $\mathrm{C}$ & 30.8 & 10.0 & 4.7 & $83 \pm 4$ & 1759 & 2.7 \\
\hline $\mathrm{D}$ & 41.1 & 9.4 & 4.2 & $84 \pm 5$ & 1276 & 2 \\
\hline $\mathrm{E}$ & 61.7 & 8.7 & 3.5 & $95 \pm 6$ & 975 & 1.5 \\
\hline $\mathrm{F}$ & 123.4 & 8.0 & 2.8 & $106 \pm 6$ & 1057 & 0.86 \\
\hline G & 185.1 & 7.6 & 2.4 & $133 \pm 8$ & 1081 & 0.72 \\
\hline $\mathrm{H}$ & 310.2 & 7.2 & 2.1 & $119 \pm 7$ & 1081 & 0.38 \\
\hline
\end{tabular}

micrograph for film $\mathrm{G}$ is shown in Figure 2(b) as an example of the columnar microstructure of the films. Figure 3 shows average grain sizes of the $\mathrm{W}$ films as a function of layer thickness. The grain size generally increases with thickness, ${ }^{23}$ ranging from 74 to $133 \mathrm{~nm}$, but is not proportional to layer thickness. The ratio of grain size to thickness varies from $7: 1$ for the thinnest film to $\sim 0.4: 1$ for the thickest film. The lack of a linear relationship between grain size and film thickness should aid the quantitative separation of grain size and surface contributions to the resistivity size effect. ${ }^{9,24}$
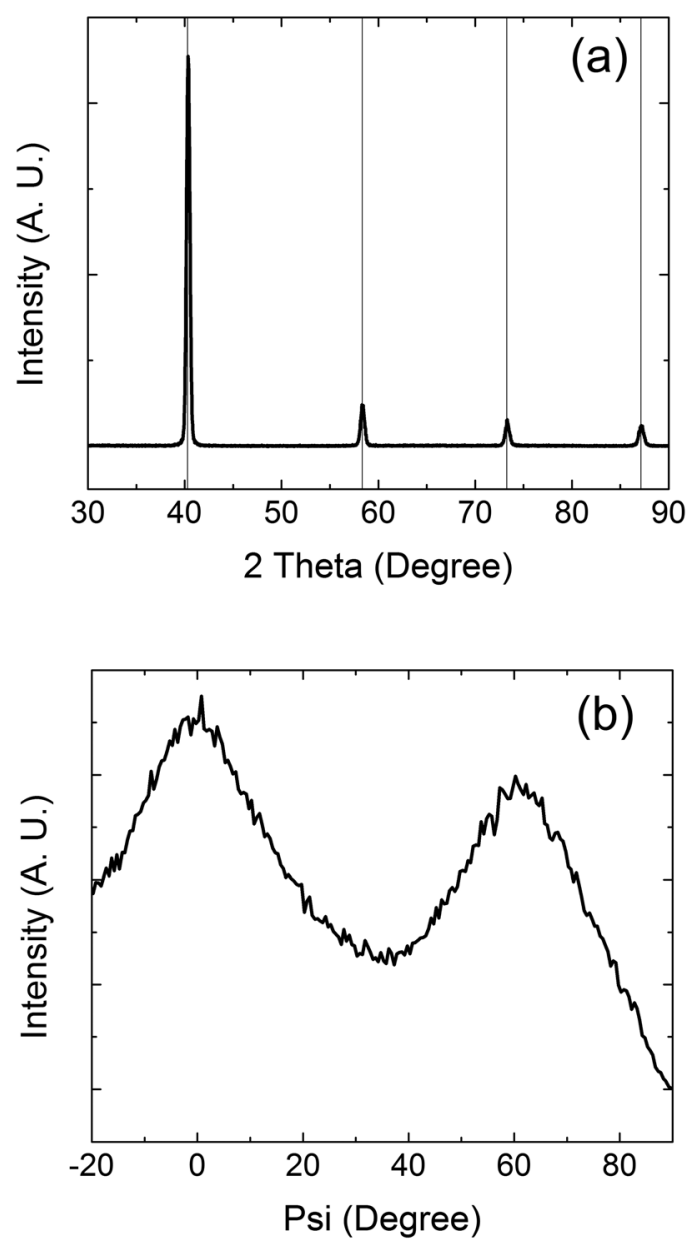

FIG. 1. (a) $\theta-2 \theta$ XRD pattern for film G. The vertical lines from left to right mark the Bragg peak positions of (110), (200), (211), and (220) planes of bcc W, respectively; (b) psi scan with respect to the W-(110) peak for film G.
The FS model was found to severely underestimate the experimentally observed film resistivity, suggesting a significant role played by grain-boundary scattering. To quantify the surface and grain boundary contributions to the resistivity size effect, the FS + MS model (Eq. (1) and (2)) and MSS model (Eq. (3)) were used, and the values of $p$ and $R$ were varied in steps of 0.01 and the corresponding sum squared error (SSE) values were calculated. This approach allows the global minimum of SSE to be determined with greater accuracy than the typical fitting approach. ${ }^{9,12}$ Using Eqs. (1)-(3), it should be noted that the $\mathrm{W}$ sputtering target used in this study was only 99.95\% pure, and thus impurities, in addition to phonons, may contribute to the background scattering. To account for this additional scattering, the resistivity of a $300 \mathrm{~nm}$ thick single crystal film similarly annealed and deposited from the same target was taken as the bulk resistivity at both $4.2 \mathrm{~K}$ $(1.32 \mu \Omega \mathrm{cm})$ and at room temperature $(6.32 \mu \Omega \mathrm{cm}){ }^{2}$ The EMFP values at these temperatures were then computed by taking the product $\rho_{\mathrm{o}}\langle\lambda\rangle_{\mathrm{o}}=1.01 \times 10^{-15} \Omega \mathrm{m}^{2}$ to be

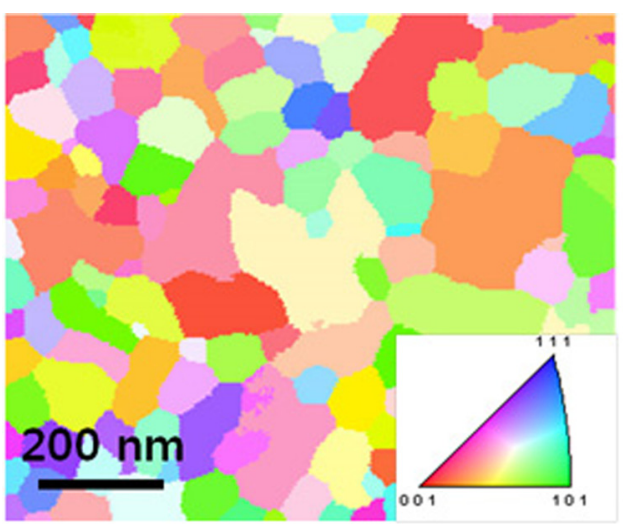

(a)

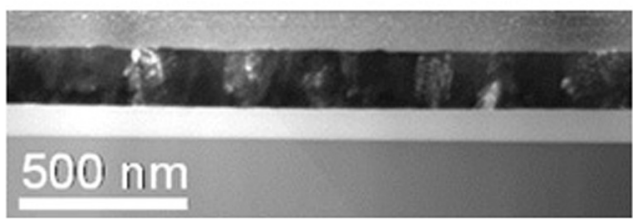

(b)

FIG. 2. (a) Representative inverse pole figure map and color key (inset) for film $\mathrm{F}$ in the sample normal direction for grain size measurement and (b) cross-sectional dark-field transmission electron micrograph of film G. 


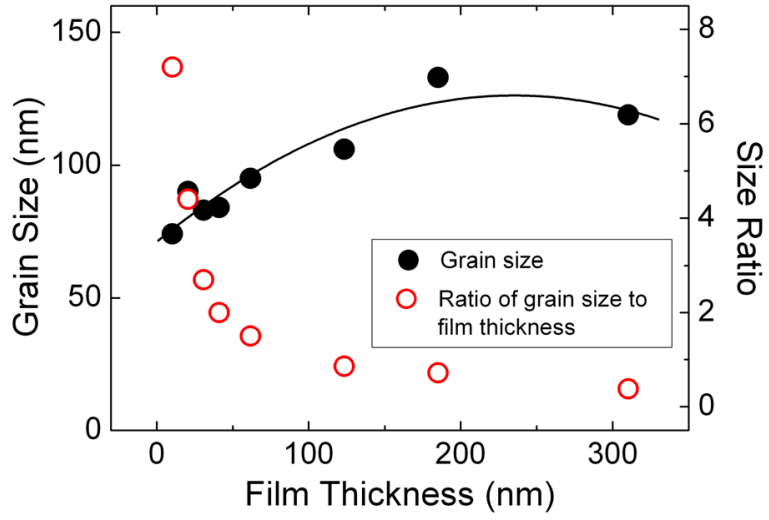

FIG. 3. Plots of grain sizes of $\mathrm{W}$ thin films and ratios of grain size to film thickness as a function of layer thickness. The second order polynomial fit for grain size as a function of film thickness is $\mathrm{y}=71.0+0.470 \mathrm{x}-0.001 \mathrm{x}^{2}$.

independent of temperature, ${ }^{2}$ which yielded $77.7 \mathrm{~nm}$ and $16.1 \mathrm{~nm}$ at 4.2 and $293 \mathrm{~K}$, respectively.

The resulting scattering parameters for the global minimum of the SSE for the FS + MS and MSS models were found to be $p=0, R=0.55$ and $p=0, R=0.56$, respectively. Table II summarizes these scattering parameters with the corresponding SSE values. The small improvement of SSE for the MSS over FS + MS and the very similar values of $p$ and $R$ for the two models indicate that these models are not distinguishable in this experiment. This result is to be contrasted with $\mathrm{Cu}$ films where a statistically significant lower SSE was observed for the FS + MS model, indicating little interaction between grain boundary and surface scattering. ${ }^{9}$ The inability of these experiments in $\mathrm{W}$ to distinguish the two models may be due to the significantly shorter EMFP of $77.7 \mathrm{~nm}$ for $\mathrm{W}$ at $4.2 \mathrm{~K}$, compared with that of $\mathrm{Cu}, 33 \mu \mathrm{m}$ at $4.2 \mathrm{~K}$. The shorter bulk EMFP for $\mathrm{W}$ is less than the grain size for all but one of the samples. Under these conditions, it is expected that isotropic scattering from impurities may mask the effect of grain-boundary scattering acting to redirect electrons toward the surfaces. Hence, the discussion hereafter will primarily focus on the results of the FS + MS model, which does not presume any direct coupling between grain-boundary and surface scattering.

Figure 4 is a contour map of the SSE values computed as functions of $p$ and $R$, using the FS + MS model. In the figure, it is clear that the changes in $R$ are accompanied with greater increases in SSE than changes of $p$. It is also noteworthy that the SSE distribution relative to the global minimum SSE is asymmetric, i.e., a rise of SSE associated with an increase in $R$ from the minimum-point $\operatorname{SSE}$ (i.e., $p=0$

TABLE II. Model parameters, $p$ and $R$, for the FS + MS model and MSS model. Numbers in the parentheses correspond to the errors at $95 \%$ confidence in the bootstrap resampling method.

\begin{tabular}{lccc}
\hline \hline Model & $p$ & $R$ & Sum squared error \\
\hline FS + MS & 0 & 0.55 & 4.6 \\
& $(+0.09)$ & $(+0.05 /-0.06)$ & 4.4 \\
MSS & 0 & 0.56 & \\
& $(+0.1)$ & $(+0.05 /-0.06)$ & \\
\hline \hline
\end{tabular}

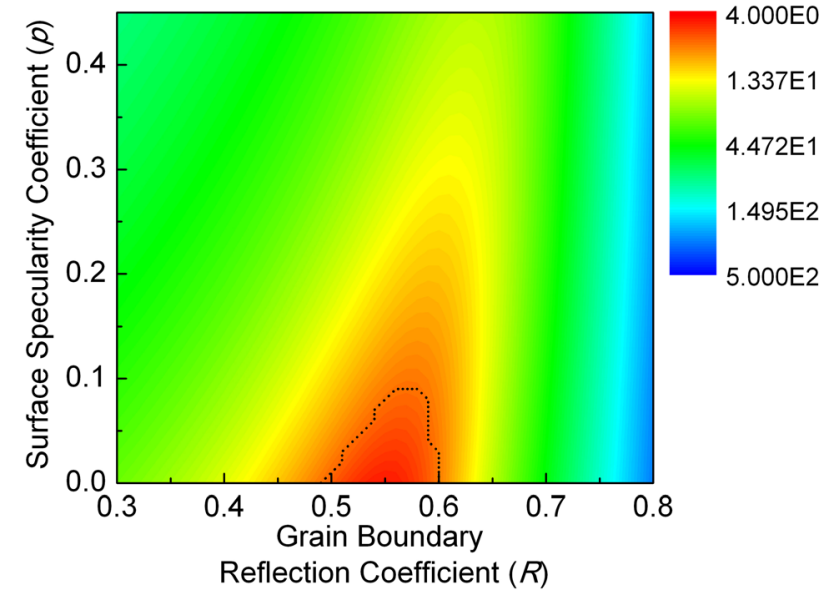

FIG. 4. Contour map of SSE as functions of $p$ and $R$, where $p$ and $R$ were varied in steps of 0.01 . The dashed curve represents limits of $p$ and $R$ at $95 \%$ confidence in bootstrap resampling.

and $R=0.55$ ) results in a greater increase in SSE than that with a decrease in $R$. In order to evaluate the reliability of $p$ and $R$ obtained by the minimization of the SSE, the error ranges were determined via the bootstrap resampling method of statistical analysis ${ }^{25,26}$ using 10000 re-samplings on the 16 SSE values to obtain the values of $p$ and $R$ at the $95 \%$ confidence level. These are summarized in Table II.

While the FS + MS can be fit to the experimental data with a clear global minimum, there are systematic errors in the fit that suggest that these models cannot be used with confidence to describe scaling of the resistivity with film thickness. This is especially true for film thicknesses below $\sim 20 \mathrm{~nm}$. In Fig. 5, the experimentally measured resistivity is subtracted from the values computed using the optimized FS + MS model and also the FS model (Eq. (1)) optimized with $p=0$. From Fig. 5, it is evident that for very thin films, the experimental resistivity is significantly larger than the model predictions, with the disagreement increasing substantially with decreasing film thickness. Comparison of the

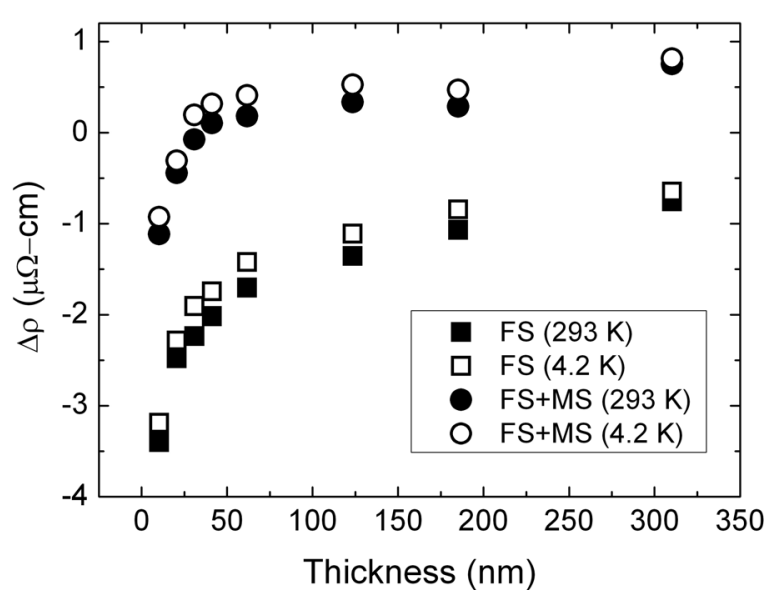

FIG. 5. Difference in resistivity, $\Delta \rho$, obtained by subtracting the experimental resistivity values from the values obtained with the FS and FS + MS models is plotted as a function of film thickness for room temperature $(293 \mathrm{~K})$ and liquid $\mathrm{He}(4.2 \mathrm{~K})$ temperature. As film thickness decreases, the models systematically predict lower resistivity, resulting in negative values for $\Delta \rho$. 
FS + MS and FS models show that while adding grainboundary scattering improves agreement with experiment, the FS + MS model has the undesirable effect of overestimating the resistivity in the limit of thick films, while simultaneously underestimating the resistivity of the thinner films. The results in Fig. 5 permit two important observations to be made. First, and most significantly, clear qualitative differences exist between the models and the experiments that cannot obviously be resolved by a different choice of model parameters. In particular, despite the assumption of fully diffuse interface scattering $(p=0)$ in both the FS + MS and FS models, the predicted resistivity is significantly lower than experimental values for the thinnest films. Possible reasons for the failure of these commonly used models to describe the experiments, along with routes for improvements of the models, are explored later in this section. The second observation is that grain-boundary scattering plays a significant role, especially for thicker films. However, the addition of grain-boundary scattering in the FS + MS model is insufficient to accurately describe the experimental data. In particular, the optimized FS + MS model results in resistivity values too large for thick films, while still remaining too small for thin films.

To underscore the importance of surface scattering in the thinner films, the predictions of the FS + MS model using the data in Tables I and II are plotted as a function of layer thickness in Fig. 6. It is shown that the surface contribution continuously increases with decreasing film thickness to become the dominant scattering mechanism at thicknesses below $20 \mathrm{~nm}$ at both 293 and $4.2 \mathrm{~K}$. This is very different from previously reported result for $\mathrm{Cu}$ films, ${ }^{9}$ where grain size was found to decrease significantly with thickness and the dominant scattering mechanism was grain boundary scattering throughout the film thickness range. The difference in behavior of $\mathrm{W}$ and $\mathrm{Cu}$ is mostly attributed to the increasingly larger ratio of grain size to film thickness (Table I) with decreasing film thickness for $\mathrm{W}$, e.g., a factor of seven for film A, whereas it is only a factor of two for $\mathrm{Cu}$ at similar dimensions. ${ }^{9,24}$ For example, if the grain size is assumed to be equal to film thickness for $\mathrm{W}$, as is generally the case for

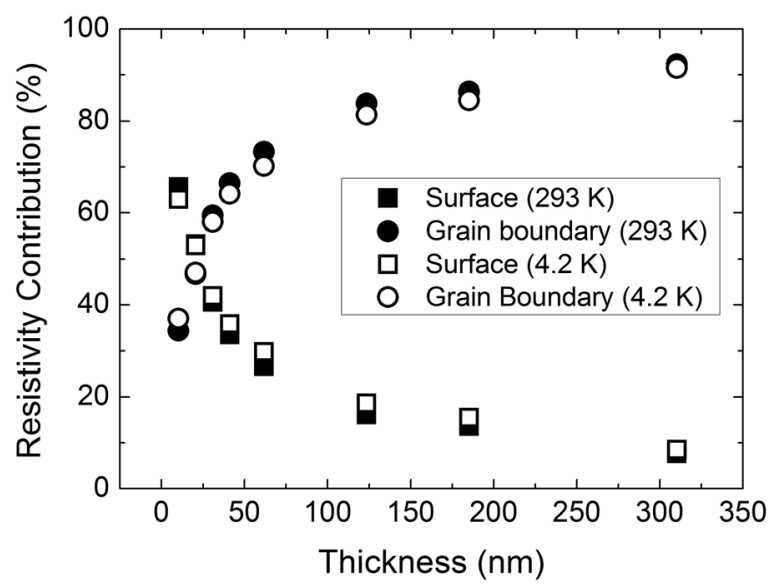

FIG. 6. Relative contributions to the resistivity size effect for surface scattering and grain boundary scattering calculated using the FS + MS model and values of $p$ and $R$ given in Table II are plotted as a function of film thickness for the experimentally observed grain sizes of samples A to $\mathrm{H}$.
$\mathrm{Cu}^{9}{ }^{9}$ calculations (not shown) reveal that the relative contribution of the grain boundary scattering is $\sim 80 \%$ in the entire thickness range in Fig. 6, which indicates that the larger grain size observed for $\mathrm{W}$, relative to film thickness, reduces the contribution of grain boundary scattering significantly.

The somewhat unusual relation between grain size and film thickness for $\mathrm{W}$ can be understood as due to a metastable $\beta$-phase that exists during film processing. It has been shown that a fine grained metastable $\beta$-phase with a grain size of $\sim 5 \mathrm{~nm}$ is formed during the initial film deposition of $\mathrm{W}$, which then transforms to a relatively coarse grained equilibrium $\alpha$-phase. ${ }^{1,14,27}$ This mechanism provides the large ratio of grain size to film thickness. Once the transformation is completed, further grain growth is limited by the low grain boundary mobility for the $\alpha-\mathrm{W}$, consistent with the modest increase in grain size with increasing film thickness observed in this study. This relatively unique grain size controlling mechanism may be a significant advantage for the use of $\mathrm{W}$ as nanoscale wires in semiconductor interconnects.

\section{BEYOND FS AND MS MODELS}

The careful experimental characterization described above allows a clear demonstration of the inability of the FS, FS + MS, or MSS models to quantitatively predict the resistivity over all temperatures and film thicknesses. This is in contrast to a recent study of $\mathrm{Cu}$ films where, while the FS model failed to describe the experiments, the FS + MS and MSS models resulted in reasonable agreement with experimental results. ${ }^{9}$ Previous studies have found similar problems in predicting the large increase in resistivity that is observed with decreasing film thickness. For example, in Refs. 28 and 29, the FS model predicts systematically lower values of the resistivity for single-crystal $\mathrm{Cu}$ films for film thicknesses below $\sim 10 \mathrm{~nm}$ even with the assumption of fully diffuse scattering $(p=0)$. This is an important observation that merits further consideration since it seems to identify a fundamental limitation of the models and brings into question the ability of the simple models of this kind to make predictions of the resistivity for even thinner films (below $10 \mathrm{~nm}$ ) where there is significant interest in determining replacement materials for $\mathrm{Cu}$ in interconnects. While there are many possible explanations for the inability of the models to describe the experiments, here we highlight a few of them with possible directions to develop more predictive theoretical models.

\section{A. Multiple relevant length scales for bulk resistivity}

Strong deviations from the FS, FS + MS, and MSS models might be due to the assumption that only one length scale, the EMFP $\lambda$, is relevant for a describing the bulk resistivity. Instead, it may be essential to consider the possibility that multiple length scales associated with electron-phonon scattering are important. This might be essential for a metal like $\mathrm{W}$, which possesses a very complex Fermi surface.

In a material with multiple bands crossing the Fermi level, each characterized by a different velocity, there should be many relevant length scales to describe electron-phonon scattering. In the case of $\mathrm{W}$, the Fermi surface is complicated, 
including the electron jack, electron ball, hole octahedron, and hole ellipsoid. ${ }^{2}$ These regions of the Fermi surface do not form a continuous surface, and the carrier velocity associated with different parts of the Fermi surface varies greatly. Improvements might be sought by developing a semiclassical model similar to existing models, but based on multiple length scales associated with bulk electron-phonon scattering occurring in different regions of the disconnected Fermi surface.

\section{B. Quantum size effects for electrons}

It is also important to remember that the FS, FS + MS, and MSS models are semiclassical models that are based on an assumption of the scattering of bulk quasiparticles. This might be expected to be reasonable when the film thickness and grain size are larger than the bulk EMFP $(L \gg \lambda$ and $g \gg \lambda)$. By contrast, in the opposite limit where $\lambda \gg L$ or $\lambda \gg g$, electrons undergo multiple interface or grain-boundary scattering events which may lead to interfering electron trajectories. As a result, in this limit, scattering events cannot be treated as independent events. The interference between multiple scattering events and different electron trajectories in a disordered system might lead to enhanced scattering, increased resistivity, diffusive transport, or even electron localization.

For thin films, the formation of minibands might also be relevant. In the instance that minibands are relevant, conductivity is predicted to exhibit a saw-tooth dependence on film thickness $L{ }^{30,31}$ Estimates of the spacing between the highest minibands is on the order $E_{f}(a / L)$, where $E_{f}$ is the Fermi energy, $a$ is the lattice parameter, and $L$ is the film thickness. For the present case, we estimate that this spacing is comparable to $k_{B} T$ at room temperature for $L \sim 10 \mathrm{~nm}$. For $\mathrm{T}=4.2 \mathrm{~K}$, the spacing between even the lowest minibands is less than $k_{B} T$, and quantum size effects could possibly be observed. Another important aspect is electron-phonon scattering, which may tend to result in smearing of the quantum size effects.

One approach to studying quantum size effects is to use first-principles calculations with Green's functions. For example, in Ref. 32, transport in small (less than 1800 atom) $\mathrm{Cu}$ films was computed using Green's functions, with the results fit to FS models. However, the resistivity was only computed for $\mathrm{T}=0 \mathrm{~K}$, and hence electron decoherence effects were not included. Another issue is the rather small sizes of the films along the conduction direction in Ref. 32, which makes comparison to experimental measurements in films with very large areas questionable. However, firstprinciples methods based on Green's functions approaches represent an important way forward towards developing more predictive models.

\section{Quantum size effects for phonons}

Finally, we consider the possibility that the formation of phonon minibands might result in size-dependent electronphonon scattering rates. Assuming linear dispersion, the frequency spacing between neighboring phonon minibands can be estimated from $\Delta \omega \sim \frac{v \pi}{L}$, where $v$ is the acoustic phonon velocity, corresponding to the energy $\hbar \Delta \omega \approx \frac{h v}{2 L}$, where $h$ is Planck's constant. For a W film with $L \sim 10 \mathrm{~nm}$, this energy is $\sim 10^{-3} \mathrm{eV}$. This is much smaller than $k_{B} T$ at room temperature, but only slightly less than $k_{B} T$ for $\mathrm{T}=4.2 \mathrm{~K}$. It seems likely that phonon miniband formation associated with very thin films could be relevant for $\mathrm{T}=4.2 \mathrm{~K}$ but not for room temperature. Moreover, the spacing between electronic minibands is larger than this scale, as described above, and probably modification of the phonon spectrum for thin films is a less significant effect.

\section{SUMMARY}

The impact of surface scattering and grain boundary scattering to the resistivity size effect in $\mathrm{W}$ films was studied using a series of polycrystalline $\mathrm{W}$ films prepared by dc sputtering. The Fuchs-Sondheimer surface scattering and Mayadas-Shatzkes grain boundary scattering models were employed for quantitative analyses. Using these models, the scattering parameters that minimized the SSE were $p=0+0.1, R=0.55 \pm 0.06$, where the error ranges were determined using the bootstrap resampling method at $95 \%$ confidence. The relative contribution of the surface scattering continuously increased with decreasing film thickness and eventually became the dominant scattering mechanism at thickness below $20 \mathrm{~nm}$, due to the modest decrease in grain size with decreasing film thickness.

Detailed comparison of the predicted resistivities with the experimental results revealed systematic deviations. Possible reasons for the inadequacy of the FS, FS + MS, and MSS models were presented: these include the possibility of more than one relevant length scale for bulk electron scattering, neglect of electron interference including mini-band formation or electron localization effects, and neglect of sizedependence of the phonon spectrum relevant for electronphonon scattering. Directions for improvement of theoretical models were presented.

\section{ACKNOWLEDGMENTS}

Financial support from the Alessandro and Piermaria Reggiori Fellowship, the Bertucci Graduate Fellowship, the Department of Materials Science and Engineering at Carnegie Mellon University, Intel Corporation, and SRC Tasks $1292.008,2121.001$, and 2323.001 is acknowledged. P.K.S. was supported in part by a grant from the Semiconductor Research Corporation.

${ }^{1}$ D. Choi, B. Wang, S. Chung, X. Liu, A. Darbal, A. Wise, N. T. Nuhfer, K. Barmak, A. P. Warren, K. R. Coffey, and M. F. Toney, J. Vac. Sci. Technol., A 29, 051512 (2011).

${ }^{2}$ D. Choi, C. S. Kim, D. N. S. Chung, A. P. Warren, N. T. Nuhfer, M. F. Toney, K. R. Coffey, and K. Barmak, Phys. Rev. B 86, 045432 (2012).

${ }^{3}$ D. Choi, M. Moneck, X. Liu, S. J. Oh, C. R. Kagan, K. R. Coffey, and K. Barmak, Sci. Rep. 3, 2591 (2013).

${ }^{4}$ C. S. Hau-Riege, Microelectron. Reliab. 44, 195 (2004).

${ }^{5}$ D. Josell, S. H. Brongersma, and Z. Tökei, Annu. Rev. Mater. Res. 39, 231 (2009).

${ }^{6}$ K. Fuchs, Proc. Cambridge Philos. Soc. 34, 100 (1938).

${ }^{7}$ E. H. Sondheimer, Adv. Phys. 1, 1 (1952).

${ }^{8}$ A. F. Mayadas and M. Shatzkes, Phys. Rev. B 1, 1382 (1970).

${ }^{9}$ T. Sun, B. Yao, A. P. Warren, K. Barmak, M. F. Toney, R. E. Peale, and K. R. Coffey, Phys. Rev. B 81, 155454 (2010).

${ }^{10}$ A. J. Learn and D. W. Foster, J. Appl. Phys. 58, 2001 (1985).

${ }^{11}$ I. P. Ivanov, I. Sen, and P. Keswick, J. Vac. Sci. Technol. B 24(2), 523 (2006). 
${ }^{12}$ G. M. Mikhailov, A. V. Chernykh, and V. T. Petrashov, J. Appl. Phys. 80(2), 948 (1996).

${ }^{13}$ W. Steinhögl, G. Schindler, G. Steinlesberger, M. Traving, and M. Engelhardt, J. Appl. Phys. 97, 023706 (2005).

${ }^{14}$ S. M. Rossnagel, I. C. Noyan, and C. Cabral, Jr., J. Vac. Sci. Technol. B 20, 2047 (2002).

${ }^{15}$ E. Fawcett and D. Griffiths, J. Phys. Chem. Solids 23, 1631 (1962).

${ }^{16}$ V. E. Startsev, V. P. D’yakina, V. I. Cherepanov, N. V. Volkenshtein, R. Sh. Nasyrov, and V. G. Manakov, Zh. Eksp. Teor. Fiz. 79, 1335 (1980) [Sov. Phys. JETP 52, 675 (1980)].

${ }^{17}$ L. J. van der Pauw, Philips Res. Rep. 13, 1 (1958).

${ }^{18}$ E. F. Rauch, J. Portillo II, S. Nicolopoulos, D. Bultreys, S. Rouvimov, and P. Moeck, Z. Kristallogr. 225, 103-109 (2010).

${ }^{19}$ E. F. Rauch and M. Veron, Materialwiss. Werkstofftech. 36, 552 (2005).

${ }^{20}$ A. D. Darbal, K. J. Ganesh, X. Liu, S. B. Lee, J. Ledonne, T. Sun, B. Yao, A. P. Warren, G. S. Rohrer, A. D. Rollett, P. J. Ferreira, K. R. Coffey, and K. Barmak, Microsc. Microanal. 19, 111 (2013).
${ }^{21}$ X. Liu, D. Choi, H. Beladi, N. T. Nuhfer, G. S. Rohrer, and K. Barmak, Scr. Mater. 69, 413 (2013).

${ }^{22}$ D. T. Carpenter, J. M. Rickman, and K. Barmak, J. Appl. Phys. 84, 5843 (1998).

${ }^{23}$ C. V. Thompson, Annu. Rev. Mater. Sci. 30, 159 (2000).

${ }^{24}$ T. Sun, B. Yao, A. P. Warren, K. Barmak, M. F. Toney, R. E. Peale, and K. R. Coffey, Phys. Rev. B 79, 041402 (2009).

${ }^{25}$ B. Efron, Ann. Stat. 7, 1 (1979).

${ }^{26}$ B. Efron, Stat. Sci. 18, 135 (2003).

${ }^{27}$ I. C. Noyan, T. M. Shaw, and C. C. Goldsmith, J. Appl. Phys. 82(9), 4300 (1997).

${ }^{28}$ E. T. Krastev, L. D. Voice, and R. G. Tobin, J. Appl. Phys. 79, 6865 (1996).

${ }^{29}$ J. M. Purswani and D. Gall, Thin Solid Films 516, 465 (2007).

${ }^{30}$ S. Chatterjee and A. E. Meyerovich, Phys. Rev. B 81, 245409 (2010).

${ }^{31}$ N. Trivedi and N. Ashcroft, Phys. Rev. B 38, 12298 (1988).

${ }^{32}$ Y. Ke, F. Zahid, K. Xia, D. Gall, and H. Guo, Phys. Rev. B 79, 155406 (2009). 